\title{
Time degenerate Neumann initial boundary value problems and applications
}

\author{
Dhaigude R.M., Sandip M., Patil Devkatte and Bodkhe S.G.* \\ Department of Mathematics, Arts, Science and Commerce College, Badnapur, 431202, MS, \\ Sandip Nivas, Vidhut Nagar, Nanded, 431602, MS \\ *Department of Statistics Arts, Science and Commerce College, Badnapur, 431202, MS, India, \\ rmdhaigude@gmail.com, sgbodkhe@gmail.com
}

\begin{abstract}
The aim of this paper is to develop monotone method for nonlinear time degenerate parabolic Neumann initial boundary value problems. These Problems are important in the Study of Physics, Ecology, Heat and Mass Transfer and any other branches of science and Engineering. Monotone Method is one of the important method in the theory of differential equations The main idea of this method is to develop iterative scheme based on the notion of upper and lower solutions. Using upper and lower solutions as distinct initial iterations, two monotone sequences are constructed. It is shown that these two sequences converge monotonically from about and below to maximal and minimal solutions respectively which lead to the existence - comparison and uniqueness results for the solution of nonlinear time degenerate Neumann IBVP. Positivity lemma is also obtained which play an important role in the proofs of these results.

Key words- Neumann IBVP, positivity Lemma, Upper and Lower solution, Monotone Property, Existence comparison and uniqueness solutions
\end{abstract}

\section{Introduction}

The method of upper and lower solution is constructive and powerful method employed successfully in the study of partial differential equations. In this method maximal and minimal solutions play an important role. The existence of pair of distinct maximal and minimal solutions for parabolic boundary value problems was first time observed by Mlak [3]. In 1972 Sattinger [5] has first time developed the method of upper and lower solutions for nonlinear parabolic as well as elliptic boundary value problems. Since then his work refined and extended by many researchers and series of papers appeared in the literature. An excellent account of these results is given in the agant books by Ladde, Lakshmikantham and Vatsala [2] and Pao [4]. Here we develop monotone scheme for Neumann initial boundary value problems. The content of the paper is as follows:

In section 2, we prove Positivity Lemma. The notion of upper and lower solution is defined Section 3, is devoted for monotone scheme. Applications for Neumann initial boundary value problems are discussed in the last section.

\section{Positivity of Solutions}

Consider the time degenerate Neumann initial boundary value problem (IBVP)

$\mathrm{d}(\mathrm{x}, \mathrm{t}) \mathrm{u}_{\mathrm{t}}-\mathrm{D}(\mathrm{x}, \mathrm{t}) \nabla^{2} \mathrm{u}=\mathrm{f}(\mathrm{x}, \mathrm{t}, \mathrm{u})$; in $\mathrm{D}_{\mathrm{T}}$

Boundary condition $\frac{B}{b V} \mathrm{u}(\mathrm{x}, \mathrm{t})=\mathrm{h}(\mathrm{x}, \mathrm{t})$; on $\mathrm{S}_{\mathrm{T}}$

Initial condition $\mathrm{u}(\mathrm{x}, 0)=\mathrm{u}_{0}(\mathrm{x})$; $\quad$ in $\Omega$, Where $\Omega$ is a bounded domain is $R^{p}(\mathrm{p}=1,2, \ldots$. with boundary $\partial \Omega$, $\mathrm{D}_{\mathrm{T}}=\Omega \times(0, \mathrm{~T}), \mathrm{S}_{\mathrm{T}}=\partial \Omega \times(0, \mathrm{~T}), \mathrm{T}>0$, Assume that (i) The coefficient $d(x, t)$ is nonnegative in $D_{T}$. However we will not assume that $d(x, t)$ is bounded away from zero, Since $d(x, t)=0$ for some $(x, t)$ $\in D_{T}$ and hence the equation is time degenerate,

(ii) $\mathrm{D}(\mathrm{x}, \mathrm{t})>0$ in $\mathrm{D}_{\mathrm{T}}$,

(iii) $\mathrm{f}(\mathrm{x}, \mathrm{t}, \mathrm{u}), \mathrm{h}(\mathrm{x}, \mathrm{t})$ and $\mathrm{u}_{0}(\mathrm{x})$ are Holder continuous function in their respective domains

(iv) $f(x, t, u)$ satisfies the Lipschitz condition $u$ in $D_{T}$.

$$
-\mathrm{d}(\mathrm{x}, \mathrm{t})\left(\mathrm{u}_{1}-\mathrm{u}_{2}\right) \leq \mathrm{f}\left(\mathrm{x}, \mathrm{t}, \mathrm{u}_{1}\right)-\mathrm{f}\left(\mathrm{x}, \mathrm{t}, \mathrm{u}_{2}\right) \leq \overline{\mathrm{c}}(\mathrm{x}, \mathrm{t})\left(\mathrm{u}_{1}-\mathrm{u}_{2}\right),
$$

for $u_{1}, u_{2} \in(\hat{u}, \tilde{u})$

LEEMA 2.1 [Positivity Lemma] : suppose that $\mathrm{w} \in \mathrm{C}\left(\overline{\mathrm{D}}_{\mathrm{T}}\right) \mathrm{I} \mathrm{C}^{2,1}\left(\mathrm{D}_{\mathrm{T}}\right)$ and satisfies the inequalities

(i) $d(x, t) w_{t}-D(x, t) \nabla^{2} w+c(x, t) w \geq 0$; in $D_{T}$

(ii) $\alpha_{0}(\mathrm{x}) \frac{\partial \mathrm{w}}{\partial \mathrm{v}}+\beta(\mathrm{x}) \mathrm{w} \geq 0 ; \quad$ on $\mathrm{S}_{\mathrm{T}}$ (2.3)

(iii) $w(x, 0) \geq 0$; in $\Omega$ where $d=d(x, t) \geq 0, c(x, t) \geq 0$, in $D_{\text {T. }}$. Then $\mathrm{w}(\mathrm{x}, \mathrm{t}) \geq \emptyset$ in $\overline{D_{T}}$.

PROOF : Proof is very simple so details are omitted.

Now we define upper and lower solutions of the time degenerate Neumann IBVP (2.1)

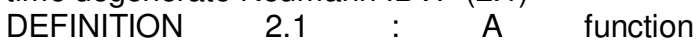
U e $\mathrm{C}\left(\overline{\mathrm{D}}_{\mathrm{T}}\right) \cap \mathrm{C}^{2,1}\left(\mathrm{D}_{\mathrm{T}}\right)$ is called an upper solution of the time degenerate parabolic Neumann IBVP(2.1) if it satisfies the inequalities 


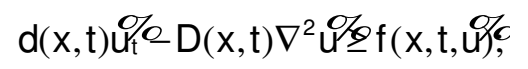

in

$\mathrm{D}_{\mathrm{T}}$

$$
\frac{3}{3 v} \mathrm{u}(\mathrm{x}, \mathrm{t})=\mathrm{h}(\mathrm{x}, \mathrm{t}) ; \text { on } \mathrm{S}_{\mathrm{T}}(2.4)
$$

$\mathrm{U}(\mathrm{x}, 0) \geq \mathrm{u}_{0}(\mathrm{x})$; in $\Omega$

DEFINITION $2.2 \quad: \quad$ A function $\hat{\mathrm{u}} \in \mathrm{C}\left(\overline{\mathrm{D}_{\mathrm{T}}}\right) \cap \mathrm{C}^{2,1}\left(\mathrm{D}_{\mathrm{T}}\right)$ is called a lower solution of the time degenerate parabolic Neumann IBVP (2.1) if it satisfies the inequalities

$d(x, t) \hat{u}_{t}-D(x, t) \nabla^{2} \hat{u} \leq f(x, t, \hat{u}) ;$ in $D_{T}$

$\frac{d}{3 v} \mathrm{u}(\mathrm{x}, \mathrm{t})=\mathrm{h}(\mathrm{x}, \mathrm{t}) ;$ on $\mathrm{S}_{\mathrm{T}}(2.5)$

$\hat{u}(x, 0) \leq u_{0}(x) ;$ in $\Omega$

DEFINITION 2.3 : The functions $\tilde{u}, \hat{u}$ are called ordered upper and lower solutions if $\tilde{u} \geq \hat{u}$

in $\overline{\mathrm{D}_{\mathrm{T}}}$,

DEFINITION 2.4 : For any ordered upper and lower solutions $\tilde{u}, \hat{u}$ the sector is denoted by $(\tilde{u}, \hat{u})$ and is defined as

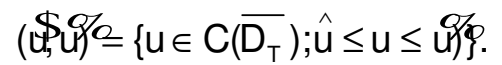

\section{Monotone Iteration Scheme}

Consider the following iteration scheme with suitable initial iteration $\mathrm{u}^{(0)}$

$\mathrm{d}(\mathrm{x}, \mathrm{t}) \mathrm{u}_{\mathrm{t}}^{(\mathrm{k})}-\mathrm{D}(\mathrm{x}, \mathrm{t}) \quad \nabla^{2} \mathrm{u}^{(\mathrm{k})}+\underline{\mathrm{c}}(\mathrm{x}, \mathrm{t}) \mathrm{u}^{(\mathrm{k})}=\underline{\mathrm{c}} \quad(\mathrm{x}, \mathrm{t}) \mathrm{u}^{(\mathrm{k}-}$

1) $+\mathrm{f}\left(\mathrm{x}, \mathrm{t}, \mathrm{u}^{(\mathrm{k}-1)}\right)$; in $\mathrm{D}_{\mathrm{T}}$

$\frac{d}{3 V} u^{(\mathrm{k})}(\mathrm{x}, \mathrm{t})=\mathrm{h}(\mathrm{x}, \mathrm{t}) ;$ on $\mathrm{S}_{\mathrm{T}}(3.1)$

$u^{(k)}(x, 0)=u_{0}(x), \ln \Omega$

For $\mathrm{k}=1$, we have

$d(x, t) u_{t}^{(1)}-D(x, t) \nabla^{2} u^{(1)}+c(x, t) u^{(1)}=6(x, t) u^{(0)}+f\left(x, t, u^{(0)}\right)$;

in $\mathrm{D}_{\mathrm{T}}$

$\frac{a}{3 v} u^{(1)}(x, t)=h(x, t) ;$ on $S_{T}$

$$
u^{(1)}(x, 0)=u_{0}(x)
$$

in $\Omega$

Since $u^{(0)}$ is known, the R.H.S. is known. The existence theory for linear time degenerate parabolic Neumann initial boundary value problem implies that $u^{(1)}$ exists.

Similarly for $\mathrm{k}=2$, we have.

$$
\begin{array}{r}
d(x, t) u_{t}^{(2)}- \\
\text { in } \mathrm{D}_{\top}
\end{array}
$$

$\frac{3}{a v} u^{(2)}(x, t)=h(x, t) ; \quad$ on $\mathrm{S}_{\mathrm{T}}$

$$
\mathrm{u}^{(2)}(\mathrm{x}, 0)=\mathrm{u}_{0}(\mathrm{x}) \text {; in } \Omega
$$

Since $u^{(1)}$ is known the R.H.S. is known, The existence theory for nonlinear time degenerate parabolic Neumann initial boundary value problem implies that $u^{(2)}$ exists. Thus for $k=3,4$ ,$\ldots$. We get $u{ }^{(3)}, u^{(4)}, \ldots$. Thus we construct a sequence $\left(u^{(1)}, u^{(2)}, u,{ }^{(3)} \ldots.\right)$ we denote it by $\left\{u^{(k)}\right\}$. The sequence $\left\{u^{(k)}\right\}$ so obtained is well defined.

We choose initial iteration $u^{(0)}=\tilde{u}$ the sequence so obtained is donated by $\left\{\bar{u}^{(k)}\right\}$ We choose initial iteration $u^{(0)}=\mathcal{U}_{\text {}}$, the sequence so obtained is denoted by $\left\{\bar{u}^{(k)}\right\}$. Thus choosing an upper solution or lower solution as the initial iteration, we get upper and lower sequences $\left\{\bar{u}^{-(k)}\right\}$ and $\left\{\underline{u}^{(k)}\right\}$ respectively.

\section{LEMMA 3.1 [Monotone Property]: Suppose} that

(i) $\tilde{u}, \hat{u}$ are ordered upper and lower solutions of time degenerate Neumann IBVP

$d(x, t) u_{t}-D(x, t) \nabla^{2} u=f(x, t, u) ;$ in $\mathrm{D}_{\mathrm{\top}}$

$\frac{\partial}{2 v} \mathrm{u}(\mathrm{x}, \mathrm{t})=\mathrm{h}(\mathrm{x}, \mathrm{t})$; on $\mathrm{S}_{\mathrm{T}}$

$u(x, 0)=u_{0}(x)$; in $\Omega$

(ii) $f$ satisfies the one sided Lipschitz condition

$f\left(x, t, u_{1}\right)-f\left(x, t, u_{2}\right) \geq-\underline{c}(x, t)\left(u_{1}-u_{2}\right)$,

for $\mathrm{u}_{1}, \mathrm{u}_{2} \in(\hat{\mathrm{u}}, \tilde{\mathrm{u}})$.

Then the sequence $\left(u^{(k)}\right),\left(\underline{u}^{(k)}\right)$ possess the monotone property

$\hat{\mathrm{u}} \leq \underline{\mathrm{u}}^{(\mathrm{k})} \leq \underline{\mathrm{u}}^{(\mathrm{k}+1)} \leq \overline{\mathrm{u}}^{(\mathrm{k}+1)} \leq \overline{\mathrm{u}}^{(\mathrm{k})} \leq \boldsymbol{\mathrm { U }}$ \% $\overline{\text { in } \overline{\mathrm{D}_{\mathrm{T}}}}$

Moreover $\bar{u}^{(k)}$ and $\underline{u}^{(k)}$ are ordered upper and lower solutions of time degenerate Neumann IBVP for every $\mathrm{k}=12$, 3,.

PROOF :- Define

$$
\begin{aligned}
& \mathrm{w}=\overline{\mathrm{u}}^{-(0)}-\overline{\mathrm{u}}^{(1)} \\
& \mathrm{w}=\mathrm{U}^{-} \mathrm{u}^{-(1)},\left(\mathrm{Q} \overline{\mathrm{u}}^{(0)}=\mathrm{u}\right)^{\mathrm{k}}
\end{aligned}
$$

Since UYis an upper solution, we have by definition 2.1 $u^{(1)}+f\left(x, t, u^{(1)}\right)$; $\mathrm{d}(\mathrm{x}, \mathrm{t}) \tilde{\mathrm{u}}_{\mathrm{t}}-\mathrm{D}(\mathrm{x}, \mathrm{t}) \nabla^{2} \tilde{\mathrm{u}} \geq \mathrm{f}(\mathrm{x}, \mathrm{t}, \tilde{\mathrm{u}})$; in $\mathrm{D}_{\mathrm{T}}$

$$
\frac{\partial}{i v} \vec{u}(\mathrm{x}, \mathrm{t})=\mathrm{h}(\mathrm{x}, \mathrm{t}) ; \text { on } \mathrm{S}_{\mathrm{T}}
$$


$\tilde{u}(x, 0) \geq \tilde{u}_{0}(x)$; in $\Omega$

Clearly,

$d(x, t) w_{t}=d(x, t) \tilde{u}_{t}-d(x, t) \bar{u}_{t}^{(1)}$ and

$-D(x, t) \nabla^{2} w=-D(x, t) \nabla^{2} \tilde{u}+D(x, t) \nabla^{2} \bar{u}^{-(1)}$

$\underline{c}(x, t) w=\underline{c}(x, t) \tilde{u}-\underline{c}(x, t) \nabla^{2} \bar{u}^{(1)}$

By adding we get

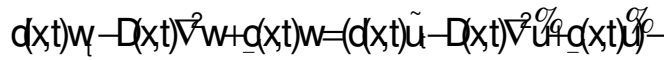

$\left(\mathrm{d}(\mathrm{x}, \mathrm{t}) \overline{\mathrm{u}}_{\mathrm{t}}^{(1)}-\mathrm{D}(\mathrm{x}, \mathrm{t}) \nabla^{2} \overline{\mathrm{u}}^{(1)}+\underline{\mathrm{c}}(\mathrm{x}, \mathrm{t}) \overline{\mathrm{u}}^{-(1)}\right)$

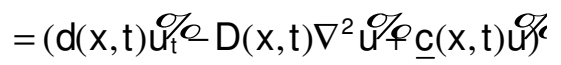

$-\left(\underline{c}(x, t) u^{-(0)}+f\left(x, t, u^{\phi \theta}\right)\right.$

(By iterative process)

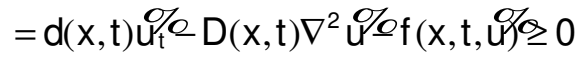

$\therefore \mathrm{d}(\mathrm{x}, \mathrm{t}) \mathrm{w}_{\mathrm{t}}-\mathrm{D} \nabla^{2} \mathrm{w}+\underline{\mathrm{c}}(\mathrm{x}, \mathrm{t}) \mathrm{w} \geq 0$.

Also,

$$
\begin{gathered}
\frac{\partial}{i v} w=\frac{\partial}{\partial v} \tilde{u}-\vec{u}^{(1)} \\
\frac{\partial}{i n} w=\frac{\partial}{i v} \tilde{u}-h(x, t) \geq 0
\end{gathered}
$$

And

$\mathrm{w}(\mathrm{x}, 0)=\tilde{\mathrm{u}}(\mathrm{x}, 0)=\mathrm{u}_{0}(\mathrm{x}) \geq 0$.

Now applying the lemma 2.1 we get

$w(x, t) \geq 0 \quad$ in $\overline{\mathrm{D}}_{\mathrm{T}}$. (3.5)

This implies that

$\bar{u}^{-(1)} \leq \bar{u}^{-(0)}$

We also know that $\hat{u}$ is a lower solution.

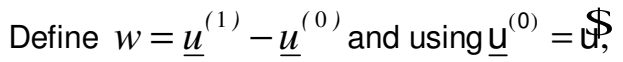

We have. $w=\underline{u}^{(1)}-\$$.

Using the above argument, we get

$\underline{u}^{(1)} \geq \underline{u}^{(0)}$ (3.6)

Next we define

$$
w^{(1)}=\bar{u}^{(1)}-\underline{u}^{(1)}
$$

Clearly,

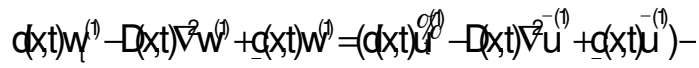

$\left(\mathrm{d}(\mathrm{x}, \mathrm{t}) \underline{\mathrm{u}}_{\mathrm{t}}^{(1)}-\mathrm{D}(\mathrm{x}, \mathrm{t}) \nabla^{2} \underline{\mathrm{u}}^{(1)}+\underline{\mathrm{c}}(\mathrm{x}, \mathrm{t}) \underline{\mathrm{u}}^{(1)}\right)$

$\left.=\mid f\left(x, t, \underline{u}^{-(0)}\right)+\underline{q}(x, t) \bar{u}^{(0)}\right]-\left[f\left(x, t, \underline{u}^{(0)}\right)+\underline{q}(x, t) \underline{u}^{(0)}\right]$

(using iterative process)

$$
=\underline{c}(x, t)(\tilde{u}-\hat{u})+f(x, t, \tilde{u})-f(x, t, \hat{u}) \geq 0
$$

$\left(\bar{u}^{(0)}=\tilde{u}, \underline{u}^{(0)}=\hat{u} \quad\right.$ and using Lipschiz condition (2.2))

$\mathrm{d}(\mathrm{x}, \mathrm{t}) \mathrm{w}_{\mathrm{t}}^{(1)}-\mathrm{D}(\mathrm{x}, \mathrm{t}) \nabla^{2} \mathrm{w}^{(1)}+\underline{\mathrm{c}}(\mathrm{x}, \mathrm{t}) \mathrm{w}^{(1)} \geq 0$.

Also,

$$
\begin{aligned}
& \frac{\partial}{i v} w^{(0)}=h(x, t)-h(x, t)=0 \\
& w^{0}(x, 0)=u_{0}(x)-u_{0}(x)=0
\end{aligned}
$$

Applying the Lemma 2.1 we get,

$w^{(1)} \geq 0$ (3.7)

This shows that

$\underline{u}^{(1)} \leq \bar{u}^{(1)}$

Thus we conclude that

$\underline{u}^{(0)} \leq \underline{u}^{(1)} \leq \bar{u}^{(1)} \leq \bar{u}^{(0)}$.

Assume by induction

$\underline{u}^{(k-1)} \leq \underline{u}^{(k)} \leq \bar{u}^{(k)} \leq \bar{u}^{(k-1)}$ in $\overline{D_{T}}$ (3.9)

Define function

$w^{(k)}=\bar{u}^{(k)}-\bar{u}^{(k+1)}$

Using iterative process and Lipschitz condition, $w^{(k)}$ satisfies the relation

$\mathrm{d}(x, t) w_{t}^{(k)}-\mathrm{Q}(x, t) \nabla^{2} w^{(k)}+\underline{q}(x, t) w=\left[f(x, t, \bar{u})^{(k-1)}+\underline{q}(x, t) u^{-(k-1)}\right]$ $-\left\lfloor f\left(x, t, \bar{u}^{(k)}\right)+\underline{c}(x, t) u^{-k}\right\rfloor \geq 0 ;$

$\mathrm{n} \mathrm{D}_{\mathrm{T}}$

$$
\frac{a}{i n} w(x, t)=0
$$

$w^{(k)}(x, 0)=0 ;$ in $\Omega$

on $\quad \mathrm{S}_{\mathrm{T}}$

Again applying the Lemma 2.1 we get

$w^{(k)} \geq 0$

i.e. $\underline{u}^{(k+1)} \leq \bar{u}^{(k)} \cdot(3.10)$

On similar lines

$\underline{u}^{(k+1)} \leq \underline{u}^{(k)}$

and $\quad \underline{u}^{(k+1)} \leq \bar{u}^{-(k+1)}$

Thus we have, from the principle of induction

$\hat{\mathrm{u}} \leq \underline{\mathrm{u}}^{(\mathrm{k})} \leq \underline{\mathrm{u}}^{(\mathrm{k}+1)} \leq \overline{\mathrm{u}}^{(\mathrm{k}+1)} \leq \overline{\mathrm{u}}^{(\mathrm{k})} \leq \mathcal{u}$ in $\overline{D_{T}}$

for every $\mathrm{k}=1,2,3, \ldots$.

Hence the result.

\section{APPLICATONS}

In this section it is shown that this powerful method is useful in obtaining Existence comparison and Uniqueness results for parabolic 
Neumann initial boundary value problem with linear boundary condition.

Theorem 4.1 [Existence - comparison

Theorem]

Suppose that

(i) $\tilde{u}, \hat{u}$ are the ordered upper and lower solutions of Neumann IBVP

$\mathrm{d}(\mathrm{x}, \mathrm{t}) \mathrm{u}_{\mathrm{t}}-\mathrm{D}(\mathrm{x}, \mathrm{t}) \nabla^{2} \mathrm{u}=\mathrm{f}(\mathrm{x}, \mathrm{t}, \mathrm{u})$; in $\mathrm{D}_{\mathrm{T}}$

$$
\frac{\partial}{i n v} u(x, t)=F(x, t) \text { i on } S_{T}
$$

$u(x, 0)=u_{0}(x) ;$ in $\Omega$

(ii) $f$ satisfies the one sided Lipschitz condition

$f\left(x, t, u_{1}\right)-f\left(x, t, u_{2}\right) \geq-d(x, t)\left(u-u_{2}\right), f o r u \hat{u} u_{2} \leq u_{1} \leq \tilde{u}(x, t) \in \bar{D}$.

Then, the sequence $\left\{\bar{u}^{-(k)}\right\},\left\{\underline{u}^{(k)}\right\}$ converges monotonically to unique solution $u$ of time degenerate Neumann IBVP (2.1) and satisfy the relation,

$\mathbb{\mathrm { U }} \leq \underline{\mathrm{u}}^{(1)} \leq \ldots \leq \underline{\mathrm{u}} \leq \overline{\mathrm{u}} \leq \ldots \leq \overline{\mathrm{u}}^{(1)} \leq \overline{\mathrm{u}}^{(1)} \leq \mathcal{U}, \boldsymbol{O}$ in $\overline{\mathrm{D}_{\mathrm{T}}}$ (4.1)

PROOF : We show the monotone convergence of the maximal and minimal sequences $\left\{\bar{u}^{-(k)}\right\}$ and $\left\{\underline{u}^{(k)}\right\}$ respectively Suppose $w=\bar{u}^{(0)}-\bar{u}^{(1)}$, where $\bar{u}^{(0)}-\underline{u}$. By using the definition 2.1 and iterative system we have,

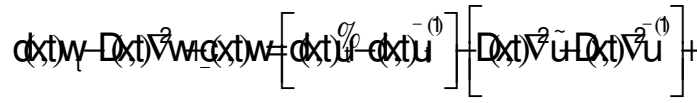
$\underline{\mathrm{c}}(\mathrm{x}, \mathrm{t}) \tilde{\mathrm{u}}-\underline{\mathrm{c}}(\mathrm{x}, \mathrm{t})-\overline{\mathrm{u}}^{(1)}$

$=\left[\mathrm{d}(\mathrm{x}, \mathrm{t}) \tilde{\mathrm{u}}_{\mathrm{t}}-\mathrm{D}(\mathrm{x}, \mathrm{t}) \nabla^{2} \tilde{\mathrm{u}}+\underline{\mathrm{c}}(\mathrm{x}, \mathrm{t}) \tilde{\mathrm{u}}\right]$
$-\left\lfloor d(x, t) \bar{u}_{t}^{(1)}-D(x, t) \nabla^{2} \bar{u}^{(1)}+\underline{c}(x, t) \bar{u}^{(1)}\right\rfloor$ $=\left[\mathrm{d}(x, \mathrm{t}) \tilde{\mathrm{u}}-\mathrm{d}(x, \mathrm{t}) \nabla^{2} \tilde{\mathrm{u}} \underline{\mathrm{q}}(\mathrm{x}, \mathrm{t}) \tilde{\mathrm{u}}\right]-\left[\underline{\mathrm{g}}(\mathrm{x}, \mathrm{t}) \overline{\mathrm{u}}^{-(0)}+\mathrm{f}\left(\mathrm{x}, \mathrm{t}, \mathrm{u}^{-(0)}\right)\right](\mathrm{B}$ y iterative process)

$=d(x, t) \tilde{u}_{t}-D(x, t) \nabla^{2} \tilde{u}-f(x, t, \tilde{u}) \geq 0$

$\therefore \mathrm{d}(\mathrm{x}, \mathrm{t}) \mathrm{w}_{\mathrm{t}}-\mathrm{D}(\mathrm{x}, \mathrm{t}) \nabla^{2} \mathrm{w}+\underline{\mathrm{c}}(\mathrm{x}, \mathrm{t}) \mathrm{w} \geq 0$.

Also, $w=\tilde{u}-\bar{u}^{(1)}$

$\frac{\partial}{i v} w=\frac{a}{i v} \quad \ddot{\omega}-h(x, t) \geq 0$

and $w(x, 0)=\tilde{u}(x, 0)-u_{0}(x) \geq 0$
By using the Lemma 2.1 we get,

$$
\begin{aligned}
& w(x, t) \geq 0 \quad \text { in } \overline{\mathrm{D}}_{\mathrm{T}} \\
& \therefore \bar{u}^{(0)} \geq \bar{u}^{(1)}
\end{aligned}
$$

We also know that UYis a lower solution.

Define $w=\underline{u}^{(1)}-\underline{u}^{(0)}$ and using $\underline{u}^{(0)}=\hat{u}$

We get $w=\underline{u}^{(1)}-\hat{u}$

Then we obtain, $w \geq 0$. This gives $\underline{u}^{(1)} \geq \underline{u}^{(0)}$

Next suppose, $w^{(1)}=\bar{u}^{(1)}-\underline{u}^{(1)}$. Then by using the given Lipschitz condition and by iterative system we get,

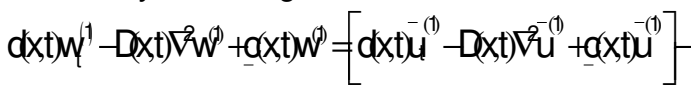

$\left[\mathrm{d}(\mathrm{x}, \mathrm{t}) \underline{\mathrm{u}}_{\mathrm{t}}^{(1)}-\mathrm{D}(\mathrm{x}, \mathrm{t}) \nabla^{2} \underline{\mathrm{u}}^{(1)}+\underline{\mathrm{c}}(\mathrm{x}, \mathrm{t}) \underline{\mathrm{u}}^{(1)}\right]$

$=\left[\underline{c}(x, t) u^{-(0)}+f\left(x, t, u^{-(0)}\right)\right]-\left[f\left(x, t, \underline{u}^{(0)}\right)+\underline{c}(x, t) \underline{u}^{(1)}\right]$

(using iterative process)

$=\underline{c}(x, t)(\tilde{u}-\hat{u})+f(x, t, \tilde{u})-f(x, t, \hat{u}) \geq 0$

$\left(\bar{u}^{(0)}=\tilde{u}, \underline{u}^{(0)}=\hat{u}\right.$ and by Lipschitz condition $)$

$\mathrm{d}(\mathrm{x}, \mathrm{t}) \mathrm{w}_{\mathrm{t}}^{(1)}-\mathrm{D}(\mathrm{x}, \mathrm{t}) \nabla^{2} \mathrm{w}^{(1)}+\underline{\mathrm{c}}(\mathrm{x}, \mathrm{t}) \mathrm{w}^{(1)} \geq 0$.

Also,

$\frac{\partial}{r^{2} v} w^{0}=h(x, t)-h(x, t)=0$

$w^{(1)}(x, 0)=u_{0}(x)-u_{0}(x)=0$

Thus by Lemma 2.1 we get,

$w^{(1)} \geq 0$

$\underline{u}^{(1)} \leq \bar{u}^{(1)}$

Thus (4.2), (4.3) and (4.4), we get

$\underline{u}^{(0)} \leq \underline{u}^{(1)} \leq \bar{u}^{(1)} \leq \bar{u}^{-(0)}$

Assume that

$\underline{u}^{(k-1)} \leq \underline{u}^{(k)} \leq \bar{u}^{(k)} \leq \bar{u}^{(k-1)}$; in $\bar{D}_{T}$.

Suppose

$w^{(k)}=u^{-(k)}-u^{-(k+1)}$

Then we obtain $w^{(k)} \geq 0$

So $\quad u^{(k)} \geq \bar{u}^{(k+1)}$

Similarly, consider (4.5)

$\mathrm{w}^{(\mathrm{k})}=\underline{\mathrm{u}}^{(\mathrm{k}+1)}-\underline{\mathrm{u}}^{(\mathrm{k})}$. 
We obtain $w^{(k)} \geq 0$

$\therefore \underline{\mathrm{u}}^{(\mathrm{k}+1)} \geq \underline{\mathrm{u}}^{(\mathrm{k})}$. (4.6)

Letting,

$w^{(k)}=\bar{u}^{(k+1)}-\underline{u}^{(k+1)}$

$\therefore \bar{u}^{(\mathrm{k}+1)} \geq \underline{\mathrm{u}}^{(\mathrm{k}+1)}$.

Thus from (4.5), (4.6), and (4.7), we have,

$\underline{\mathrm{u}}^{(\mathrm{k})} \leq \underline{\mathrm{u}}^{(\mathrm{k}+1)} \leq \overline{\mathrm{u}}^{(\mathrm{k}+1)} \leq \overline{\mathrm{u}}^{(\mathrm{k})}$. (4.8)

Thus monotone property 3.4 follows from principle of mathematical induction.

Now we conclude that the sequence $\left\{\underline{u}^{(k)}\right\}$ is monotone nonincreasing and is bounded from below hence it is convergent. Also the sequence $\left\{\underline{u}^{(k)}\right\}$ is monotone nondecreasing and is bounded from above. Hence it is convergent.

So,

$$
\lim _{k \rightarrow \infty} \bar{u}^{(k)}=\bar{u}(x, t) \quad \text { and }
$$

$\lim _{k \rightarrow \infty} \underline{u}^{(k)}(x, t)=\underline{u}(x, t)$ exists.

and are called maximal and minimal solutions respectively of the time degenerate parabolic Neumann initial boundary value problem (2.1) and they satisfy the monotone property,

$\hat{\mathrm{u}} \leq \underline{\mathrm{u}}^{(1)} \leq \underline{\mathrm{u}}^{(2)} \leq \ldots \leq \underline{\mathrm{u}} \leq \overline{\mathrm{u}} \leq \ldots \leq \overline{\mathrm{u}}^{-(2)} \leq \overline{\mathrm{u}}^{(1)} \leq \tilde{u}$. Hence the result.

Theorem 4.2 [Uniqueness Theorem] : Suppose that

(i) $\tilde{u}, \hat{u}$, are the ordered upper and lower solution of Robin IBVP (2.1),

(ii) $f(x, t, u)$ satisfies the Lipschitz condition on (2.2)

Then Robin IBVP (2.1) has unique solution.

Proof : We know that $\bar{u}(x, t), \underline{u}(x, t)$ are maximal and minimal solutions respectively of the time degenerate IBVP (2.1)

To Prove uniqueness we suppose that,

$w(x, t)=\bar{u}(x, t)-\underline{u}(x, t)$

$\therefore d(x, t) w_{t}-D(x, t) \nabla^{2} w+f(x, t, \bar{u})-f(x, t, \underline{u})$

$\geq-\underline{\mathrm{c}}(\mathrm{x}, \mathrm{t})(\overline{\mathrm{u}}(\mathrm{x}, \mathrm{t})-\underline{\mathrm{u}}(\mathrm{x}, \mathrm{t}))$

$\geq-\underline{c}(x, t) w(x, t)$

$\therefore d(x, t) w_{t}-D(x, t) \nabla^{2} w+\underline{c}(x, t) w(x, t) \geq 0$ in $\mathrm{D}_{\mathrm{T}}$

$\frac{\partial}{i n v} \mathrm{w}(\mathrm{x}, \mathrm{t})=\frac{\partial}{i v} \overline{\mathrm{u}}(\mathrm{x}, \mathrm{t})-\frac{\partial}{i n v} \underline{u}(\mathrm{x}, \mathrm{t})=\mathrm{h}(\mathrm{x}, \mathrm{t})=\mathrm{h}(\mathrm{x}, \mathrm{t})=0$

, on $\mathrm{S}_{\mathrm{T}}$

$w(x, 0)=\bar{u}(x, 0)-\underline{u}(x, 0)=u_{0}(x)-u_{0}(x)=0$ in $\Omega$
By using the positivity Lemma we get,

$w(x, t) \geq 0$

$\therefore \bar{u}(x, t) \geq \underline{u}(x, t) \quad$ in $\bar{D}_{T}(4.9)$

Also we can show that

$\bar{u}(x, t) \leq \underline{u}(x, t)(4.10)$

Inequalities (4.9) and (4.10) implies that

$\bar{u}(x, t) \equiv \underline{u}(x, t) \quad$ in $\mathrm{D}_{\mathrm{T}}$

Therefore, Neumann IBVP (2.1) has a unique solution. Hence the result.

\section{References}

[1] Ippolito P.M. (1978) J. Math. Anal. Appl. 64 ,530-561.

[2] Ladde G.S., Lakshmikantham V. and Vatsala A.S. (1985) Pitman, New Yorks, 1985.

[3] Mlak W. (1963) Ann. Polon. Math., 13, 101103.

[4] Pao C. V. (1992) Plenum Press, New York

[5] Sattinger D.H. (1972) Indiana Uni. Math. J., 21, 979-1000.

[6] Waid M.C. (1974) SIAM. J. Appl. Math. 26. 1 196-202. 